\title{
SPIN WAVES IN ANTIFERROMAGNETICALLY COUPLED MULTILAYERS
}

\author{
M. Mingozzi, O. Donzelli, J.M.V. Ngaboyisonga and F. Nizzold \\ Istituto Nazionale per la Fisica della Materia, Unità di Ferrara \\ and \\ Dipartimento di Fisica, Università di Ferrara, 44100 Ferrara, Italy
}

We investigate how the static and dynamical magnetic properties of $\mathrm{Fe} / \mathrm{Cr}$ finite superlattices with antiferromagnetic coupling depend on the ratio between the uniaxial anisotropy constant and the interlayer exchange constant of Fe films. The spin waves are calculated by considering dipole interaction, intralayer and interlayer exchange coupling, uniaxial anisotropy, in presence of an external field. The calculated spectra are compared with recent experimental Brillouin scattering data which exhibit two spin waves peaks.

PACS numbers: 75.70.-i, 75.30.Pd, 75.70.Cn

In the last ten years ferromagnetic/nonmagnetic multilayers have attracted great interest mainly because of the antiferromagnetic interlayer coupling and the giant magnetoresistance effect. In particular, these effects have been studied in $\mathrm{Fe} / \mathrm{Cr}$ systems of different type [1-3]. From the theoretical point of view, several investigations have been devoted to the study of the static and dynamical magnetic properties of antiferromagnetically coupled multilayers with a finite even numbers $N$ of ferromagnetic layers [4-7].

Within the mean field approach proposed by Mills [8], and lately adopted by several authors $[9,4-7]$, the energy of $N$ magnetic layers in an applied magnetic field $H_{0}$ is given by (in magnetic-field units):

$$
E=\frac{H_{\mathrm{E}}}{2} \sum_{l=1}^{N-1} \cos \left(\alpha_{l}+\alpha_{l+1}\right)-\frac{H_{\mathrm{A}}}{2} \sum_{l=1}^{N} \cos ^{2}\left(\alpha_{l}\right)-H_{0} \sum_{l=1}^{N} \cos \left(\alpha_{l}\right),
$$

where $l$ is the layer index and $H_{\mathrm{E}}$ and $H_{\mathrm{A}}$ (both positive) are the effective interlayer exchange coupling and the uniaxial anisotropy constant, respectively. The angle $\alpha_{l}$ between the magnetization of the $l$-th layer and the external field $\boldsymbol{H}_{0}$ is positive for clockwise rotation with $l$ odd and negative with $l$ even. Both $\boldsymbol{H}_{0}$ and the magnetization are assumed to be parallel to the surfaces of the layers. As a result of the three competing energy terms in Eq. (1), four magnetic phases exist, depending on the value of $H_{0}[4-7]$ :

(i) for low fields $\left(H_{0}<H_{\mathrm{s}}\right)$ the system is antiferromagnetically aligned; 
(ii) when $H_{0}$ becomes greater than a critical field $H_{\mathbf{s}}$ a surface spin-flop transition occurs with two nearly antiferromagnetic regions separated by a domain wall;

(iii) by increasing $H_{0}$, when $H_{\mathrm{c} 3}<H_{0}<H_{\mathrm{c} 2}$, the configuration is symmetric with respect to the centre of the multilayer $\left(\alpha_{l}=\alpha_{N-l+1}\right)$. Therefore $H_{\mathrm{c} 3}$ is a critical field for a bulk spin-flop transition;

(iv) finally, for $H_{0}>H_{\mathrm{c} 2} \approx 2 H_{\mathrm{E}}-H_{\mathrm{A}}$, the alignment is ferromagnetic.

In this paper we consider the static properties and the corresponding spin waves of the configurations (i) and (iii). We omit the case (ii) which has been extensively treated [3-7] and, in addition, exhibits discontinuous jumps of the ground state [7]. In all the cases we have investigated, the ground state of the system has been determined by minimizing the energy given by Eq. (1) following the procedure outlined by Keffer and Chow [9].

The spin waves have been calculated within a macroscopic approach, which extends the theory of Hillebrands to multilayers with canted magnetization [10], based on the partial waves expansion. The propagation wave vector $Q_{\|}$of the spin waves is normal to the applied magnetic field. The Landau-Lifschitz equation of motion of the magnetization has been solved in each layer by including dipole interaction, intralayer exchange coupling (coupling constant $A$ ) and bulk uniaxial anisotropy (strengh $K_{U}=\frac{1}{2} H_{\mathrm{A}} M_{\mathrm{s}}$ ). The values used in all cases for Fe are: $A=$ $1.6 \times 10^{-6} \mathrm{erg} / \mathrm{cm}, Q_{\|}=0.67 \times 10^{5} \mathrm{~cm}^{-1}$, saturation magnetization $4 \pi M_{\mathrm{s}}=$ $17 \mathrm{kGs}$. The thicknesses of the Fe and $\mathrm{Cr}$ films are 30 and $13 \AA$. All these values have been chosen in order to compare our calculations with the magnons spectra of Yoshihara et al. [1]. The boundary conditions include the Hoffmann equations [11], which involve the intralayer exchange coupling (strength $A$ ) and the interlayer

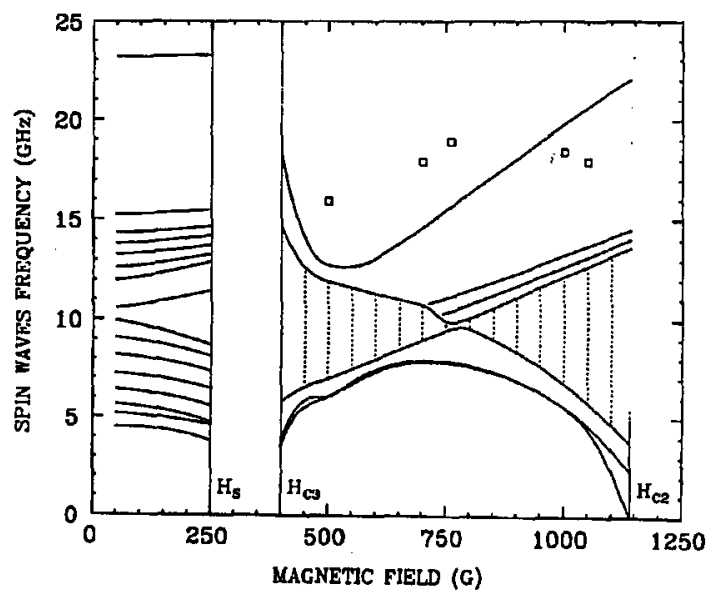

Fig. 1. Spin-wave spectrum of the 16-layer Fe/Cr superlattice described in the text, as function of the external field $H_{0}$. The dotted region represents a quasi-continuum of bulk modes. The open squares are experimental data from light scattering measurements [1]. The effective field constants are $H_{\mathrm{A}}=0.14 \mathrm{kGs}$ and $H_{\mathrm{E}}=0.645 \mathrm{kGs}$. 

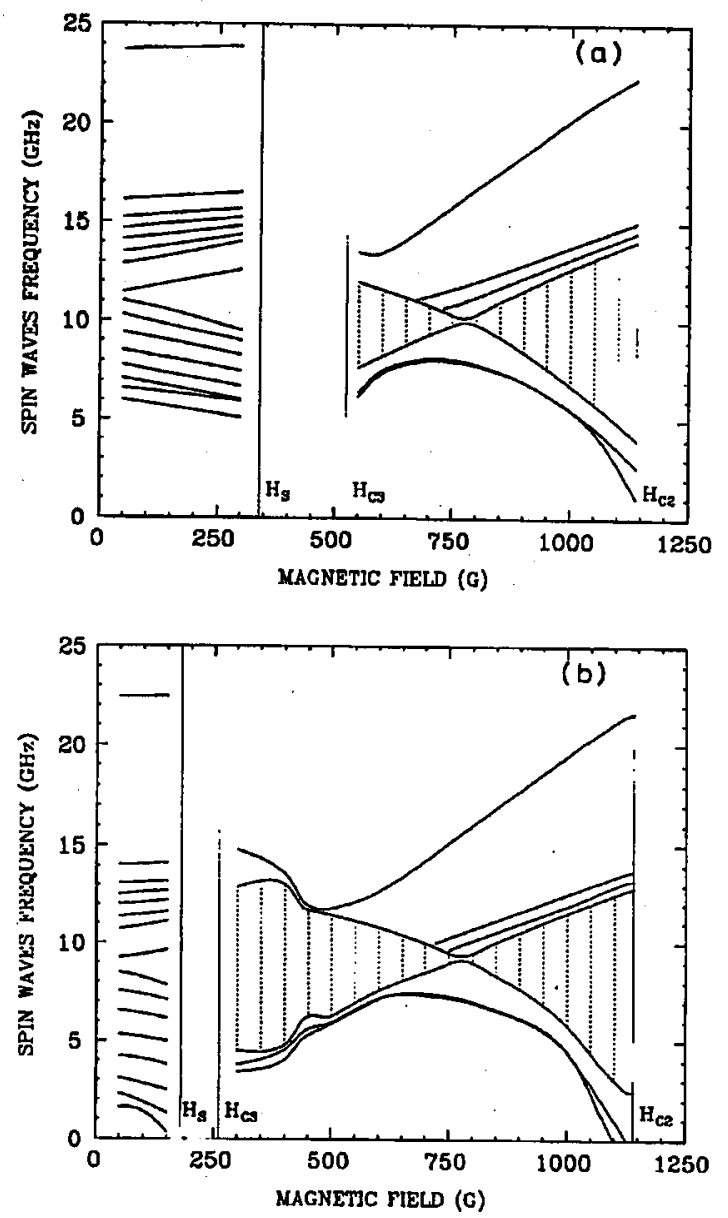

Fig. 2. The same as in Fig. 1, except: $I_{\mathrm{A}}=0.242 \mathrm{kGs}$ and $H_{\mathrm{E}}=0.691 \mathrm{kGs}$ (a), and $H_{\mathrm{A}}=0.06 \mathrm{kGs}$ and $H_{\mathrm{E}}=0.6 \mathrm{kGs}(\mathrm{b})$.

exchange coupling (strength $A_{12}=-\frac{1}{4} H_{\mathrm{E}} M_{\mathrm{s}} d_{\mathrm{Fe}}$ ). No interface anisotropy has been included. Other numerical values used in the calculation (see figure captions) have been chosen in order to have a fixed saturation field $H_{\mathrm{c} 2}=1.14 \mathrm{kGs}$ [1].

In Fig. 1 we show the calculated spin waves frequencies for $H_{\mathrm{A}} / H_{\mathrm{E}}=0.217$, which corresponds to $H_{\mathrm{c} 3}=0.4 \mathrm{kGs}$, in agreement with the measured hysteresis loop [1]. Figure 2 shows the spin wave spectra with $H_{\mathrm{A}} / H_{\mathrm{E}}=0.10$ and 0.35 , respectively. In general, the outcome of our calculations is close to the results of Wang and Mills [5], who used a different approach for the spin waves, where the magnetization was kept constant inside each layer and the interlayer dipolar interaction was explicitly taken into account. In our framework this interaction is indirectly introduced through the boundary conditions. In the interesting region of symmetric canted magnetization $H_{\mathrm{c} 3}<H_{0}<H_{\mathrm{c} 2}$, we find a moderate lowering of the mode frequencies as the ratio $H_{\mathrm{A}} / H_{\mathrm{E}}$ is decreased. 
The comparison between theory and experiment is shown in Fig. 1. Yoshihara et al. [1] found in the field region 0.5-1.05 kGs a reciprocal Brillouin peak in the high frequency range 16-19 GHz. We believe that the experimental points in the range $0.5-0.75 \mathrm{kGs}$ must be assigned to the upper calculated mode in Fig. 1 which, in this range of $H_{0}$, is localized on both the superlattice surfaces and therefore is detected in Stokes and anti-Stokes spectra. At higher fields this mode becomes a true surface mode and disappears from the Brillouin spectra (taken in grazing incidence). We assign the two experimental peaks close to $1 \mathrm{kGs}$ in Fig. 1 to the other modes split off from the bulk band. However, in order to fit the data, calculated modes with frequencies larger than those reported in Fig. 1 of about $4 \mathrm{GHz}$ would be required, indicating that additional effects, like surface anisotropy and/or biquadratic interlayer coupling, could give a better quantitative agreement. These effects are presently under investigation.

Work partially supported by MURST and CNR (contract 93.01316 ).

\section{References}

[1] A. Yoshihara, K. Takanashi, Y. Obi, H. Fujimori, J. Magn. Magn. Mater. 126, 333 (1993).

[2] E.E. Fullerton, M.J. Conover, J.E. Mattson, C.H. Sowers, S.D. Bader, Phys. Rev. $B$ 48, 15755 (1993).

[3] R.W. Wang, D.L. Mills, E.E. Fullerton, S. Kumar, M. Grimsditch, Phys. Rev. B 53, 2627 (1996).

[4] R.W. Wang, D.L. Mills, E.E. Fullerton, J.E. Mattson, S.D. Bader, Phys. Rev. Lett. 72, 920 (1994).

[5] R.W. Wang, D.L. Mills, Phys. Rev. B 50, 3931 (1994).

[6] L. Trallori, P. Politi, A. Rettori, M.G. Pini, J. Villain, Phys. Rev. Lett. 72, 1925 (1994).

[7] L. Trallori, P. Politi, A. Rettori, M.G. Pini, J. Villain, J. Phys., Condens. Matter 7, L451 (1995).

[8] D.L. Mills, Phys. Rev. Lett. 20, 18 (1968).

[9] F. Keffer, H. Chow, Phys. Rev. Lett. 31, 1061 (1973).

[10] B. Hillebrands, Phys. Rev. B 41, 530 (1990).

[11] F. Hoffmann, Phys. Status Solidi 41, 807 (1970). 